\title{
Long-term follow-up of patients with neurocysticercosis and the development of seizures
}

\author{
Fandiño-Franky Jaime*1, Guerra-Olivares Randy ${ }^{1}$, Piña-Cabrales Sandra ${ }^{1}$, Fandiño-MerzJavier ${ }^{2}$, Mayor Liliana ${ }^{2}$,Tellez Carlos Alfonso ${ }^{2}$, \\ Merlano-Zabaleta Alfonso ${ }^{3}$, Vitola Bacter ${ }^{3}$, Jiménez JD ${ }^{3}$, García $\mathrm{CE}^{3}$ and Rodríguez Y Tomás ${ }^{3}$ \\ ${ }^{1}$ Colombian Foundation Center for Epilepsy and Neurological Diseases,FIRE, Cartagena, Colombia \\ ${ }^{2}$ Medicine doctor in Social Service, Colombia \\ ${ }^{3}$ Research Group in epilepsy, Colombia
}

\begin{abstract}
Neurocysticercosis (NCC) is one of the most common causes of new onset seizures in young adults and even in infants; it is also one of the most important infectious etiologies of the central nervous system in developing countries, causing long-term sequelae such as epilepsy, dementia and hydrocephalus. To evaluate clinical cases of neurocysticercosis (NCC), correlating entry symptoms, epidemiological history, diagnostic methods, imaging, antigenic titles of ELISA (enzyme linked inmunosorvent) in serum and cerebrospinal fluid (CSF) and long-term sequelae caused by infection. A descriptive, chronological, 1744 NCC cases registered at the institution under hospital management with the same protocol since 1989 until 2012, with clinical inclusion criteria, imaging, epidemiological and laboratory; drug treatment with antihelmintics, corticosteroids and anticonvulsants; EEG monitoring with images and control of 3 , 6 and 12 months after treatment. Total distribution of $58.7 \%$ male, $75.3 \%$ of cases diagnosed before 40 years of age, a mean age of 29.08 for men, 25.96 for women. Entry symptoms: Seizures (91.68\%), headaches (4.58\%) and focal deficit signs (1.83\%). Type of seizure: Focal with secondary generalization (45.60\%) and tonic-clonic generalized. Positive ELISA in serum (61.46\%) and (36.41\%) in CSF respectively ( $\mathrm{p}=0.047)$ TAC was positive in $74.13 \%$ of cases. MRI 25.86\% and EEG were abnormal in 45.9\%. Localization: intraparenchimatous multiple: $40.99 \%$ of cases. $26.20 \%$ in frontal lobe and $21.27 \%$ in parietal lobe. Lesion type: $34.74 \%$ vesicular with scolex, $19.5 \%$ coloid. $6.76 \%$ calcified cyst and $21.27 \%$ mixed forms. Four or more lesions were associated with Intraparenchimatous multiple. 20.6\% acquired recurrent crisis (epilepsy) (p<05). In our setting, the NCC in developing countriesis a major cause of epilepsy patients within working age and even in infancy; it continues to be an endemic problem of regional health therefore the direct costs are not only from medical and surgical management, but the consequences have alsoa negative socio-economic impact. Early diagnosis and treatment limit the appearance of complications and late sequelae such as symptomatic epilepsy. The burden of epilepsy for families is very important.
\end{abstract}

\section{Introduction}

Neurocysticercosis (NCC) or larval stage of Taenia Solium is a helminthiasis given by the parasite mostly known as tapeworm, whose asexual larva infests the human central nervous system. The main reservoir for its transmission is the pig who acts as an intermediate host. The definitive host is the human, by eating undercooked meat from pork, contaminated water and food, and even auto-infection and poor hygiene (Fecal-oral transmission) [1,2].

The NCC is the most common cause of de novo epilepsy in younger persons under 20 years of age within the community and of late-onset seizures in developed countries, increasing its current prevalence in the U.S. and Europe due to the migratory phenomenon. In endemic areas it affects the $25 \%$ of the population, and is responsible for about 50,000 deaths per year worldwide [3-6].

The NCC has a heterogeneous clinical course from asymptomatic infection (where the parasite dies without treatment) to various clinical stages depending on 3 factors: the shape, location and extent of infection. Seizures are the main cardinal symptom and occur in up to $90 \%$ of patients due to cyst degeneration and its peri-lessional inflammatory reaction along with other symptoms such as headache, dizziness and signs of focal deficit [7-9].

The initial stage presents viable cysts without inflammatory reaction, protected by the blood-brain barrier (BBB) and is able to evade the immune response by 4 to 5 years. In some cases, the cellular immune response destroys the cyst making its content denser until collapsing and disintegrating resulting in a calcification. Some others are located in the subarachnoid space (27-56\%) or in the ventricular system (15-20\%), being these forms associated with a higher morbidity and mortality rate [10-13].

The larva parasite is a small marginal nodule within a fluid filled cyst in vesicular stage, of similar density to the one from the cerebrospinal fluid (CSF). During the colloidal stage, the cyst begins to degenerate, causing edema and contrast enhancement in the imaging studies. The nodule stage shows some lesions that are retractable and mineralized, being lower the intensity of the inflammatory reaction. The final stage is the calcification, when the parasite is dead and appears hyperdense on CT and hypointense on MRI [14-16].

Not all NCC are inevitably epileptogenic, there are cases with episodic host inflammatory response to the residual antigen, which is intermittently recognized by the receptor even during the calcified

Correspondence to: Jaime Fandiño-Franky, Fundación Centro Colombiano de Epilepsia y Enfermedades Neurológicas, FIRE,Calle 1 ${ }^{\text {a }}$, El Edén, Barrio Ternera, Cartagena - Colombia AA 604 y 5007, Fax: 57-6618127; E-mail: fandinojaime@gmail.com

Key words: neurocysticercosis, epilepsy, parasits, ELISA, antihelmintics

Received: August 15, 2015; Accepted: September 27, 2015; Published: September 30, 2015 
stage. In a review done by Lázaro LF et al., The NCC was not involved in epileptogenic process and was rather an incidental finding in patients with mesial temporal lobe epilepsy, with complete cessation of seizures after resective surgery [14].

The MRI has proven to be more effective than CT scan in patients with parasites located at the base of the brain, brain stem, intraventricular and spinal but has limitations for detecting calcified cysts where CT appears to be more sensible [13,17]. A current method used for the immunological diagnosis of NCC is the ELISA (enzyme linked immunosorbent) and western blott in both serum and CSF[918].

Treatment of NCC is still controversial and should be individualized depending on the anatomical location of the parasites, stage, size, and number of cysts, degree of inflammation, and the severity of associated symptoms [19]. Albendazole type antihelminticsat $15 \mathrm{mg} / \mathrm{kg} /$ day for 7-15 days or praziquantel $50 \mathrm{mg} / \mathrm{kg} /$ day for 15 days have been used, the first one being of a lower cost, more cestocidal activity, greater penetration into the CSF and best plasma levels reached by adding corticosteroids such as dexamethasone. However, high unit monodoses of praziquantel (at $100 \mathrm{mg} / \mathrm{kg}$ ) are well tolerated with the benefit of increased adhesion and therapeutic costs reduction [20-22].

In case of medical treatment failure or clinical and imaging worsening, it is necessary to try a new medical treatment with another medication. If definitively the medical treatment failure, the NCC warrants neurosurgical management such as craniotomy and/ or endoscopic removal of intraventricular or subarachnoid cysts. In addition, there is the ventricular- peritoneostomy (VP) due to hydrocephalus and stereotactic guide resection in severe cases or due to the presence of cysts located in eloquent motor areas. Recurrence of NCC has been described in up to $56 \%$ of cases, likewise the recurrence of seizures in relation to the persistence of active brain lesions [23-24].

The aim of this study was to evaluate a series of 1744 clinical cases of NCC through monitoring and follow up of a hospital management protocol performed in the Fundation Colombian Center for Epilepsy and Neurological Diseases - FIRE, from 1989 until 2012.

\section{Patients and methods}

Data were collected of 1744 cases admitted from January 1989 to February 2012 from patients registered with a diagnosis of NCC at the Colombian Foundation Center for Epilepsy and Neurological Diseases - FIRE.

The institutional treatment protocol for cerebral cysticercosis comprises:

- Hospitalization for 3 days on all patients with clinical, epidemiological and imaging evidence of neurocysticercosis admitted by the outpatient clinic.

- Implementation of lumbar puncture (CSF cytochemical and ELISA) and blood sample (serum ELISA from $1910 \mathrm{yrs}$ and cytochemical).

- Study of simple and contrast Ct-Scan and later on both CtScan and MRI during admission.

- Treatment in monotherapy with first generation antiepileptic drugs (AEDs) from the first convulsive episode with evidence of active symptomatic lesion (preferable carbamazepine).

- Implementation of dexamethasone at a rate of $4 \mathrm{mg}$ parenterally daily (I.V.) for 3 days.

- Initiation of Albendazole $15 \mathrm{mg} / \mathrm{kg} /$ day between 15 and 30 days for active lesions. Nowadays 8 days.

- Imaging and electroencephalographic monitoring and control with suspension of AED's as appropriate (Medical control in three months with Ct-Scan).The AED never can be stopped until at least 5 years in epilepsy (two or more seizures). With history of only one seizure, it can be stopped according with EEG, in two years).

The lesions were classified according to the imaging pictures:

- Cystic lesion without edema (vesicular stage).

- Active cystic lesion with high uptake to contrast and edema (colloidal phase).

- Nodular lesion.

- Calcified lesion.

The studies were performed with Ct-Scan Siemens of High Resolution and Nuclear Magnetic Resonance of 0.5 Tesla and 1.5 Tesla. Electroencephalographic recordings of 16-channel (Voyayeur brand) and 32 channels with (Nicolet Viasys equipment) collection time of 25 minutes with activation maneuvers according to international protocols. Imaging controls were performed both at the start of medication and during follow-up and the disappearance of the lesion was considered as a criteria of resolution of the NCC. ELISA antibody anticysticercus studies (both in serum and CSF) were carried out in only the beginning in the institutional laboratory and were made from 1989 with substract antigens prepared by the laboratory of the National Institute of Health in Colombia. The sensibility and specificity in the beginning with this method were not very convincing. Imagen was our gold standard.

Patients were classified according to the Del Brutto criteria (2001) [17] for neurocysticercosis based on clinical, imaging, immunological and epidemiological data. These include four layered categories:

a) Absolutes: Histological demonstration of the parasite by brain or spinal cord biopsy, cystic lesions with scolex on Ct-Scan or MRI and direct visualization of parasites in sub-retineal fundus.

b) Mayors: Highly suggestive lesions of NCC on imaging "Western Blot" positive for cysticercus antibodies, resolution of cystic lesions after treatment with praziquantel and albendazole or spontaneous resolution of small lesions.

c) Minors: NCC lesion compatible with neuroimaging, suggestive clinical manifestations, positive CSF ELISA for detection of antigens or antibodies anticysticercus and cysticercosis outside the central nervous system or.

d) Epidemiologicals: Evidence from household contact with Taeniasolium infection, individuals from endemic areas or people with history of frequent travel.

Interpretation of these criteria allows two degrees of diagnostic certainty:

1. Final diagnosis in patients who have an absolute criterion or in those who have two major plus one minor and one epidemiologic criterion.

2. Likely diagnosis in patients who have one major plus 2 minor 
criteria, which has one major, one minor and one epidemiological and those with 3 minor plus one epidemiologic .

\section{Statistical analysis}

We processed, tabulated and analyzed the 1744 NCC recorded cases from January 1989 to February 2012. Frequency analysis for descriptive studies, comparison of means, contingency tables, correlation of independent variables for normal approximations and chi-square test were performed. Alpha error probabilistic $\mathrm{P}<0.05$ with confidence interval of $95 \%$ was established by using statistical software SPSS V 17.0 Inc., Chicago for Windows.

\section{Ethical considerations}

The research approval was obtained from the Institutional Ethics Committee and it was considered a lower risk descriptive research study. National regulations and technical standards in scientific research in humans were followed according to Resolution No. 8430 of 1993 of the Ministry of Social Protection of the Republic of Colombia) and codes of ethics (Declaration of Helsinki, International Conference on Harmonization Good Clinical Practice and ICH/GCP).

\section{Results}

Of the total sample of 1744 cases with heterogeneous distribution, $58.7 \%$ are male and $41.3 \%$ female. Entry age with an average of $28.6 \pm$ 17.05 and a range of (2-89), while $84.9 \%$ of cases occurred below the age of 45 years (IC95: 82.2 to 87.2 ). $55.9 \%$ of cases were found in the group of $15-44$ years $(\mathrm{p}=0.041)$ (Table 1$)$.

The (74.2\%) of the reported cases are from the province of Bolívar, followed by Sucre $(8.74 \%)$, Cordoba (6.28\%), Atlántico (4.1\%), Cesar (3.14\%), Magdalena (2.16\%), Guajira (0.9\%) and Antioquia (0.4\%). In these cases (59.4\%) came from marginalized rural areas, while (40.5\%) came from urban centers of the various provinces of the Colombian Caribbean Coast (Table 2).

Seizures were the main presenting symptom of the disease $(91.68 \%$ of cases), followed by headache $(4.58 \%)$, signs of neurological focal deficit (1.83\%) and intracranial hypertension (1.77\%).

\section{Classification of seizures}

- Focal with secondary generalization in $(45.60 \%)$

- $\quad$ Tonic - clonic seizure (32.40\%)

- $\quad$ Focal seizures $(19.60 \%)$

- $\quad$ Tonic seizures $(1.40 \%)$

\section{Imaging findings}

$74.13 \%$ of cases were diagnosed by Ct-Scan and $25.86 \%$ by positive MRI diagnosis in patients. $(\mathrm{p}=0.045) .58 .77 \%$ of the patients had a single lesion and $41.22 \%$ multiple lesions of NCC. The number of lesions did not change significantly with increasing age at entry $(\mathrm{p}=0.07)$. The $34.74 \%$ of the images presented findings of vesicular cysts with scolex, $19.15 \%$ colloidal cysts, $18.06 \%$ in granular stage, $6.76 \%$ of calcified cysts and $21.27 \%$ of mixed forms (Table 1 ).

\section{Parasite activity}

A large percentage of cysticerci were in active phase (74.36\%), followed by transitional phase (18.69\%) and inactive - reactive $(6.88 \%)$ phase, $(\mathrm{p}=0.046)$ (Table 1).
Table 1: Descriptive Analysis: Clinical characteristics and demographic registers of patients evaluated between years 1989-2012 with clinical diagnosis of NCC (1744)

\begin{tabular}{|c|c|}
\hline$(\mathrm{N}=1744)$ & \\
\hline Male & $1025(58.7 \%)$ \\
\hline Female & $719(41.3 \%)$ \\
\hline Entry age & $28.6 \pm 17.05[1-89]$ \\
\hline \multicolumn{2}{|l|}{ Entry syntoms } \\
\hline Epileptic Crisis & $1599(91.68 \%)$ \\
\hline Focal with secundary generalization & $729(45.6 \%)$ \\
\hline GTC & $518(32.4 \%)$ \\
\hline Focal & $313(19.6 \%)$ \\
\hline Tónic & $23(1.4 \%)$ \\
\hline Otras & $16(1 \%)$ \\
\hline Headache & $80(4.58 \%)$ \\
\hline Focal deficit & $32(1.83 \%)$ \\
\hline Others & $31(1.77 \%)$ \\
\hline \multicolumn{2}{|l|}{ IntracranealHipertensión } \\
\hline Positive imagen & $2(0.11 \%)$ \\
\hline Ct-Scan & $1293(74.13 \%)$ \\
\hline MRI & $451(25.86 \%)$ \\
\hline \multicolumn{2}{|l|}{ Number of lesions in imaging } \\
\hline Single lesion & $1025(58.77 \%)$ \\
\hline Multiple lesions & $719(41.22 \%)$ \\
\hline \multicolumn{2}{|l|}{ Cisticerco phase } \\
\hline Vesicular with scolex & $606(34.74 \%)$ \\
\hline Coloidal & $334(19.15 \%)$ \\
\hline Granular & $315(18.06 \%)$ \\
\hline Calcifications & $118(6.76 \%)$ \\
\hline Mixed & $371(21.27 \%)$ \\
\hline \multicolumn{2}{|l|}{ Parasit activity } \\
\hline Activ & $1298(74.36 \%)$ \\
\hline Transitional & $326(18.69 \%)$ \\
\hline Inactiv-Reactiv & $120(6.88 \%)$ \\
\hline \multicolumn{2}{|l|}{ Localización de lesiones } \\
\hline Múltiple foci & $715(40.99 \%)$ \\
\hline \multicolumn{2}{|l|}{ Intraparenchymal } \\
\hline Frontal & $457(26.20 \%)$ \\
\hline Parietal & $382(21.90 \%)$ \\
\hline Temporal & $174(9.97 \%)$ \\
\hline Extraparenchymal & $16(0.91 \%)$ \\
\hline
\end{tabular}

\section{Antibodies anticysticercus - ELISA}

The results were positive in $61.46 \%$ of samples taken in serum and at $36.41 \%$ taken in CSF respectively $(\mathrm{p}=0.047)$. The result of ELISA did 
Table 2: Precedency and geographic distribution (Neurological Hospital-FIRE).

\begin{tabular}{|c|c|}
\hline Procedencia & \\
\hline Rural & $59.40 \%$ \\
\hline Urban & $40.60 \%$ \\
\hline Geografic distribution (provinces) & \\
\hline Bolívar & $74.20 \%$ \\
\hline Sucre & $8.70 \%$ \\
\hline Córdoba & $6.30 \%$ \\
\hline Atlántico & $4.00 \%$ \\
\hline Cesar & $3.10 \%$ \\
\hline Magdalena & $2.20 \%$ \\
\hline Guajira & $0.90 \%$ \\
\hline Antioquia & $0.40 \%$ \\
\hline
\end{tabular}

not correlate with the stage of active-inactive disease $(\mathrm{p}=0.57, \mathrm{RR}=1.0$, 95\% CI 0.97-1.03).

\section{Location of the lesions}

The multiple lesions of NCC focus in different brain regions occupied a $(40.99 \%)$ of the total of evidenced samples by imaging, followed by $(26.20 \%)$ in frontal lobe, $(21.90 \%)$ parietal and $(9.97 \%)$ temporal lobe, while the intraventricular, subarachnoidal and posterior fossa regions occupy less than $(1 \%)$ of the total sample $(\mathrm{p}=0.042)$. The number of 4 or more lesions may be associated with a poor outcome (RR 1.06, 95\% CI 1.0-1.2 $\mathrm{p}<0.05$ ); however the number of lesions was not related to the outcome of the ELISA (Table 1).

\section{Electroencephalographic findings}

The paroxysmal EEG showed a pattern of electrical activity in $45.9 \%$ of patients and was normal in tracing $54.1 \%$ of all cases. EEG analysis by age groups are similar, however there is a significant difference in the distribution attributable to the group of 1 to 14 years, where there was a higher number of positive results $(\mathrm{p}<0.05)$. The EEG results did not correlate with the number of active lesions.

\section{Treatment protocol and outcome}

Of the total cases, $(92.88 \%)$ received treatment with at least one cestocidal (Albendazole or Praziquantel + Dexamethasone), (2.86\%) did not receive antihelmintic treatment and (4.24\%) received other kind of treatment (surgical resection and/or ventricular -peritoneal shunt). The (33.1\%) of patients were diagnosed with absolute criteria for NCC and had a history of taeniasis sometime in their life. Of these (8.1\%) showed a personal history of previous seizures. However, the vast majorities of these cases (61.2\%) were diagnosed with major criteria for NCC, and reported no previous personal or family history of NCC or seizures whatsoever before admission (Table 1).

On an average, $78.6 \%$ of cases reported imaging controls made within the $1^{\text {st }}$ year after admission, and $20 \%$ after the $1^{\text {st }}$ year. The $60.9 \%$ attended respective outpatient controls within the immediate $1^{\text {st }}$ year and $32.9 \%$ reported no outpatient follow-up. The $(20.06 \%)$ of cases studied resulted with sequelae of epilepsy due to NCC $(p=0.045)$ (Table 1).

\section{Discussion}

NCC is an illness related to the parasitic transmission in communities with poor health status, humans are the only possible host responsible for the spread of the parasite eggs [25]. The results presented in this study could be extrapolated to the rest of the Colombian population and other endemic countries, where NCC is considered highly prevalent, becoming a public health problem. There is still no knowledge of the crude prevalence and incidence of Neurocysticercosis within the Colombian population. Although there are some studies described in other regions of the country, they have been evaluated under a microbiological approach describing antigenic seroprevalence rates and not having into account the utility of diagnostic criteria as proposed by Del Brutto et al. [17]. These studies are limited to report the exposure levels a person has with the antigen parasite. But it's not necessarily correlated with clinical diagnosis or under imaging and therapeutic criteria. It can't be standardized with measures and clinical observations: for example of an institutional protocol along with the frequent complications and sequels of the parasites is such as epilepsy [17]. According to the National Institute of Health of Colombia, the numbers of positive seroprevalence generally range between 0.53 and $40.19 \%$ taken from data found in studies conducted at the laboratory of parasitology [25]. In our series of cases, patients from the Caribbean region and particularly the province of Bolivar were included. This is a highly endemic area for NCC where pig farms are developed in a rudimentary form without the proper conditions of health sanitary control. (No sanitary facilities as potable and served water). A positive seroprevalence rate in serum of $61.46 \%$ was found out of the total of samples taken $[17,25]$.

The NCC is a disease related to the migratory phenomenon; in the U.S. there are few reports of recent cases in the state of Texas, in a review of 111 patients with cerebral cysticercosis in the Hispanic immigrant population [26]. The vast majority of immigrants coming from Latin America to the United States are young adults, so it is the most affected age group presenting a higher incidence during the productive ages of life, similar to the results found in these series [27].

Esquivel et al. have reported 20 cases of NCC in the city of Madrid, all attributed to human migration [28]. Fleury et al. showed that the frequency of NCC has not changed significantly in Mexico in the recent years, in fact, despite the implementation of government strategies and health policies; NCC still continues to struggle with a high prevalence of porcine cysticercosis within the rural areas [29]. 75\% of cases reported in this series were diagnosed before 45 years of age, with a mean age of $28.6 \pm 17.05$ (29.08 for men and 25.96 for women). Gender distribution was $58.7 \%$ for male and $41.3 \%$ for female group, similar to that reported in most of the reviewed literature; however Montano et al., found a higher prevalence of the disease in females, probably related to other epidemiological factors [9].

The NCC is the most common cause of "de novo" seizures in young people under 20 years in the Hispanic community in the United States [27]. In our series, the average age of onset of seizures was between 20 to 40 years, in which $45.6 \%$ are focal seizures with secondary generalization, followed by $32.4 \%$ of tonic-clonic generalized seizures compared with written reports described by Ramos et al. [16]. Some patients have only 1 or 2 seizures during the entire course of the disease, while others may present with recurrent seizures that later become long-term sequelae of symptomatic epilepsy; in our series, $57 \%$ of subjects had recurrent seizures with evidence of multiple brain lesions caused by NCC ( $\mathrm{p}=0.047)$. According to the criteria suggested 
by Del Brutto et al. $33.1 \%$ of cases met absolute criteria, and $61 \%$ major criteria [17]. The $8.1 \%$ reported a history of recurrent seizures previously informed without ever being studied by a clinical specialist/ Neurologist, emerging the big question of what was developed before, if the infection per se, or if the epilepsy was fortuitously associated to the onset of cerebral cysticercosis [30].

Kumar et al. describes $69 \%$ sensitivity and $71 \%$ specificity for ELISA in the CSF, while for Western Blot it has a sensitivity of $98 \%$ and a specificity of $100 \%$ replacing the ELISA technique for cysticercosis [6]. However, we did not obtain sufficient data for comparison in our study; this was probably due to the extensive usefulness of serum or CSF ELISA as a routine procedure done in our settings because of the lower costs that it represents to the health care system. Garcia et al. reported $95 \%$ sensitivity for Ct-Scan and a much smaller percentage for MRI [4]. $74.13 \%$ of the patients treated under protocol, were diagnosed by Ct-Scan as the imaging study of more easy access and low costs and $25.86 \%$ were diagnosed by using MRI, compared to similar findings reported in the literature $(\mathrm{p}=0.045)$.

The estimated prevalence of subarachnoid cysts has been reported between 27 to $56 \%$ and between 15 to $20 \%$ for intraventricular cysts with a high morbidity and mortality associated [31]. In contrast to our study we found only a very low prevalence of intraventricular and subarachnoidal cysts $(0.91 \%)$, which can be related to the limited access and use of special sequences in MRI studies, or we can say that in our region there are uncommon findings of extraparenchymal NCC, "theory of heterogeneity in Latin America" supported by other authors.

There are four levels of important evidence to support the hypothesis of calcified cysts and its main role in epileptogenesis:

a) The high prevalence of typical calcifications in patients with seizures or epilepsy.

b) The positive correlation between endemic populations and the increase in the proportion of seizure activity with associated calcifications.

c) The high risk of persistent seizure activity due to a solitary calcified granuloma probably related to the scolex antigen presentation intermittently with associated edema and

d) The cytotoxic role of calcium [32]. Calcifications range between $9 \%$ and $18 \%$ in randomized studies of endemic populations and up to $83 \%$ on selected studies as reported by Nash et al. This study showed a percentage of $6.76 \%$ of isolated cerebral calcifications and $21,27 \%$ of associated forms (multiple forms) as an imaging finding during routine studies (simple and contrasted TAC).

Some authors have shown the correlation between electroencephalographic (EEG) activity and localization of cerebral NCC cysts between $26-55 \%$ of cases [12,32]. However, the type of seizures seen in the interictal EEG activity did not correlate with brain location of cysts in a high percentage (54.1\%) in our report. Of the total of study subjects, $92.88 \%$ received cestocidal treatment associated with corticosteroid as part of the protocol, of which $47.8 \%$ were treated for more than 15 days and $35.8 \%$ for less than 15 days. This variation in the therapeutic regimen was due to recurrence of active cysticercosis and efficacy or abandon of treatment; but the duration of the therapeutic regimen still remains a subject of controversy, even when taking into account the monotherapy option in order to ensure therapeutic adherence [21,22].

In a study done by Carpio et al. $40.3 \%$ of subjects, including those who had seizure recurrence after treatment, showed that the recurrence rate was $22 \%$ at 6 months, $32 \%$ at 12 months, $39 \%$ at 24 months and $49 \%$ at 48 months, respectively. Treatment with an anthelmintic (Albendazole), however did not influence on recurrence. This was rather associated with persistent cysts in $56 \%$ of cases, and the risk of having another seizure after the second one was $68 \%$ with a follow-up of 6 years [30]. The percentage of seizure recurrence after a first acute symptomatic attack is high, due to NCC, but this seems to be related to the persistence of active brain lesions seen on the CT scan. Based on the imagenological disappearance of parasitic lesions, antihelmintic drugs currently used are only effective in about one third of patients with intra- parenchymal viable cysts (active cysts) [33].

Despite treatment established under the stringency of inclusion parameters, diagnostic and monitoring standardized under the same protocol, $20.06 \%$ of all patients in our series developed recurrent seizures and consequences of long term epilepsy; affecting the incidence rates of epilepsy at local and regional level.

\section{Conclusion}

The presence of suggestive symptoms of seizures in adult people under age 40 should be evaluated under strict protocols through imaging and laboratory studies looking as a probable etiology of symptomatic epilepsy in association of cerebral cysticercosis infection.

The NCC is the most common parasitic infection of the central nervous system in our country, being the Caribbean area stigmatized as a high endemicity zone for cerebral cysticercosis. This study forces us to think about government measures to control parasitic infection that affects the socio-economic indicators in general, increasing incidence rates of morbidity and mortality associated with symptomatic epilepsy $(20.06 \%)$. Colombia has prevalence in epilepsy around 20 per thousand been NCC the second cause (first neonatal hypoxia) [34-39].

\section{Intellectual property}

This document follows the regulations on intellectual property citing the name or pseudonym of the author and the title of the original works that were used as described in Act 23 of 1982, taking into account the procedures set forth herein for ensure legal security of the owners of the bibliographic material used (Decree 460 of 1995).

\section{References}

1. Goldsmith Robert, Heyneman (1995) Parasitology and tropical medicine. Manual moderno 1: 639-646.

2. Pal DK, Carpio A, Sander JW (2000) Neurocysticercosis and epilepsy in developing countries. J NeurolNeurosurg Psychiatry 68: 137-143. [Crossref]

3. Kelley R, Duong DH, Locke GE (2002) Characteristics of ventricular shunt malfunctions among patients with neurocysticercosis. Neurosurgery 50: 757-761. [Crossref]

4. Garcia HH, Gonzalez AE, Gilman RH; Cysticerosis Working Group in Peru (2003) Diagnosis, treatment and control of Taeniasoliumcysticercosis. Curr Opin Infect Dis 16: 411-419. [Crossref]

5. Nash TE, Singh G, White AC, Rajshekhar V, Loeb JA, et al. (2006) Treatment of neurocysticercosis: current status and future research needs. Neurology 67: 1120-1127. [Crossref]

6. Kumar GR (2008)Neurocysticercosis: A Pictorial Review. Infect Dis Clin Pract $16: 210-217$.

7. Rajshekhar V, Raghava MV, Prabhakaran V, Oommen A, Muliyil J (2006) Active epilepsy as an index of burden of neurocysticercosis in Vellore district, India. Neurology 67: 2135-2139. [Crossref]

8. D Ciampi de Andrade Rodrigues CL, Abraham R, Castro LH, Livramento JA, et 
al. (2010) Cognitive impairment and dementia in neurocysticercosis: a cross-sectional controlled study. Neurology 74: 1288-1295. [Crossref]

9. Montano SM, Villaran MV, Ylquimiche L, Figueroa JJ, Rodriguez S, et al. (2005) Neurocysticercosis: association between seizures, serology, and brain CT in rural Peru. Neurology 65: 229-233. [Crossref]

10. Sharma R, Bargotra R, Tandon VR, Gupta SK, Verma S, et al. (2005) Neurocysticercosis: Current Vitae. JK Science 7.

11. Psarros TG, Zouros A, Coimbra C (2003) Neurocysticercosis: a neurosurgical perspective. South Med J 96: 1019-1022. [Crossref]

12. Nash TE, Del Brutto OH, Butman JA, Corona T, Delgado-Escueta A, et al. (2004) Calcific neurocysticercosis and epileptogenesis. Neurology 62: 1934-1938. [Crossref]

13. Sáenz B, Ruíz-Garcia M, Jiménez E, Hernández-Aguilar J, Suastegui R, et al. (2006) Neurocysticercosis: clinical, radiologic, and inflammatory differences between children and adults. Pediatr Infect Dis J 25: 801-803. [Crossref]

14. LL doAmaral, RM Ferreira, AJ da Rocha, Ferreira NP (2005) Neurocysticercosis: evaluation With advanced magnetic resonance techniques and atypical forms. Top Magn Reson Imaging 16: 127-144. [Crossref]

15. Rosenfeld E (2003) Neurocysticercosis. Pediatr Infect Dis J 22: 181-182. [Crossref]

16. Ramos G, Zúñiga G, Llanos M (2004) Calcificaciones cerebrales como causa de epilepsia. Análisisetiológico y clínico-topográfico. Acta Neurol Colomb 20:134-138.

17. Del Brutto OH, Rajshekhar V, White AC Jr, Tsang VC, Nash TE, et al. (2001) Proposed diagnostic criteria for neurocysticercosis. Neurology 57: 177-183. [Crossref]

18. Garcia HH, Gonzalez AE, Gilman RH (2011) Cysticercosis of the central nervous system: how should it be managed? Curr Opin Infect Dis 24: 423-427. [Crossref]

19. Singhi P, Dayal D, Khandelwal N (2003) One week versus four weeks of albendazole therapy for neurocysticercosis in children: a randomized, placebo-controlled double blind trial. Pediatr Infect Dis J 22: 268-272. [Crossref]

20. Bang OY, Heo JH, Choi SA, Kim DI (1997) Large cerebral infarction during praziquantel therapy in neurocysticercosis. Stroke 28: 211-213. [Crossref]

21. Göngora-Rivera F, Soto-Hernández JL, González Esquivel D, Cook HJ, MárquezCaraveo C, et al. (2006) Albendazole trial at 15 or $30 \mathrm{mg} / \mathrm{kg} /$ day for subarachnoid and intraventricularcysticercosis. Neurology 66: 436-438. [Crossref]

22. Del Brutto OH, Campos X, Sánchez J, Mosquera A (1999) Single-day praziquantel versus 1-week albendazole for neurocysticercosis. Neurology 52: 1079-1081. [Crossref]

23. Neal JH (1995) An endoscopic approach to cysticercosis cysts of the posterior third ventricle. Neurosurgery 36: 1040-1043. [Crossref]

24. Husain M, Rastogi M, DK Jha, Husain N, Gupta RK (2007) Endoscopic removal of fourth ventricular transaqueductalneurocysticercosis With An angiographic catheter. Neurosurgery 60: 249-253. [Crossref]

25. Florez AC (2013)Cisticercosis in Colombia: seroprevalence study 2008-2010. Acta Neurol Colomb 29: 73-86.

26. Serpa JA, Graviss EA, Kass JS, White AC Jr (2011) Neurocysticercosis in Houston, Texas: an update. Medicine (Baltimore) 90: 81-86. [Crossref]

27. Wallin MT, Kurtzke JF (2004) Neurocysticercosis in the United States: review of an important emerging infection. Neurology 63: 1559-1564. [Crossref]

28. Esquivel A, Diaz-Otero F, Gimenez-Roldan S (2005) Growing frequency of neurocysticercosis in Madrid (Spain). Neurologia 20: 116-120. [Crossref]

29. Fleury A, Moreno García J, Valdez Aguerrebere P, de SayveDurán M, Becerri Rodríguez $\mathrm{P}$, et al. (2010) Neurocysticercosis, a persisting health problem in Mexico. PLoS Negl Trop Dis 4: e805. [Crossref]

30. Carpio A, Hauser WA (2002) Prognosis for seizure recurrence in patients with newly diagnosed neurocysticercosis. Neurology 59: 1730-1734. [Crossref]

31. Levy AS, Lillehei KO, Rubinstein D, Stears JC (1995) Subarachnoidneurocysticercosis with occlusion of the major intracranial arteries: case report. Neurosurgery 36: 183188. [Crossref]

32. Park SY,Barkovich AJ, Weintrub PS (2000) Clinical implications of calcified lesions of neurocysticercosis. Pediatr Infect Dis J 19: 581-583. [Crossref]

33. Carpio A,Fleury A, Hauser WA (2013) Neurocysticercosis: Five new things. Neurol Clin Pract 3: 118-125. [Crossref]

34. Fleury, Agnès (2013) Control of TaeniaSolium Transmission of Taeniosis and Cysticercosis in Endemic Countries: The Roles of Continental Networks of Specialists and of Local Health Authorities.

35. Gómez J, Arciniegas JE, Torres J (1977) Prevalencia de la epilepsia en Bogotá Neurología en Colombia 1: 43-48

36. Fandiño J,Pacheco G (1982) Prevalencia de la epilepsia en el departamento de Bolívar. Epilepsia en Colombia 2: 6-10

37. Fandiño J, López E, Guerrero G (1985) Prevalencia de la epilepsia en los departamentos de Bolívar, Sucrey Córdoba. Epilepsia en Colombia 5: 4-8.

38. Gonzales I, Miranda JJ, Rodriguez S, Vargas V, Cjuno A, et al. (2015) Seizures, cysticercosis and rural-to-urban migration: the PERU MIGRANT study. Trop Med Int Health 20: 546-552. [Crossref]

39. Nash TE, Mahanty S Loeb JA, Theodore WH, Friedman A, et al. (2015) Neurocysticercosis: A Natural human model of epileptogenesis. Epilepsy 56: 177-183. [Crossref]

Copyright: (C2015 Jaime FF. This is an open-access article distributed under the terms of the Creative Commons Attribution License, which permits unrestricted use, distribution, and reproduction in any medium, provided the original author and source are credited. 